07,05

\title{
Исследование мезоструктуры моногерманидов переходных металлов, синтезированных под давлением
}

\author{
(C) И.А. Сафриулина ${ }^{1,2,3}$, Е.В. Алтынбаев $1,2,3$, Е.Г. Яшина ${ }^{1,2}$, A. Heinemann ${ }^{4}$, \\ Л.Н. Фомичева ${ }^{3}$, А.В. Цвященко ${ }^{3}$, С.В. Григорьев ${ }^{1,2,3}$ \\ ${ }^{1}$ Петербургский институт ядерной фризики, НИЦ „Курчатовский институт“, \\ Гатчина, Россия \\ ${ }^{2}$ Санкт-Петербургский государственный университет, \\ Санкт-Петербург, Россия \\ ${ }^{3}$ Институт фозиики высоких давлений, \\ Троицк, Москва, Россия \\ ${ }^{4}$ Helmholtz Zentrum Geesthacht, \\ Geesthzacht, Germany \\ E-mail: irina.safiulina@gmail.com
}

(Поступила в Редакцию 14 июля 2017 г.)

\begin{abstract}
Методом малоуглового рассеяния нейтронов исследована мезоструктура моногерманидов переходных металлов $\mathrm{Mn}_{1-x} \mathrm{Co}_{x} \mathrm{Ge}$ в широком диапазоне концентраций $x=0.0-0.95$. Эти соединения были синтезированы под высоким давлением и являются метастабильными при нормальных условиях. Экспериментальные зависимости $I(Q)$, полученные для всего ряда образцов в диапазоне переданных импульсов $\left(6 \cdot 10^{-2} \mathrm{~nm}^{-1}<Q<2.5 \mathrm{~nm}^{-1}\right)$, описываются степенной зависимостью $Q^{-n}$ с показателем $n=2.99 \pm 0.02$, однозначно связанным с фрактальными свойствами исследуемой системы. Полученная зависимость свидетельствует о том, что надатомная структура образцов характеризуется наличием дефектов с пространственной организацией, описываемой фрактальной моделью с логарифмической зависимостью корреляционной функции плотности дефектов. Интересно отметить, что в изоструктурном соединении $\mathrm{FeGe}$ такие дефекты отсутствуют, то есть экспериментальные зависимости интенсивности хорошо описываются выражением $Q^{-n}$ с показателем $n=4.1 \pm 0.1$, демонстрирующим наличие кристаллитов с однородным распределением плотности внутри него и резкой границей, характеризующей поверхность.
\end{abstract}

Авторы благодарят за поддержку Российский Научный Фонд (грант № 17-1201050).

DOI: 10.21883/FTT.2018.04.45687.236

\section{1. Введение}

Моногерманиды переходных металлов с кристаллографической структурой типа В20 представляют огромный интерес благодаря особенностям своей магнитной структуры [1-11]. Геликоидальный магнитный порядок в этих соединениях, обусловлен сосуществованием сильного изотропного ферромагнитного взаимодействия и антисимметричного взаимодействия ДзялошинскогоМория (модель Бака-Йенсена $[12,13])$, вызванного нарушением инверсионной симметрии в расположении атомов в кристалле. Период спиновой спирали в них меняется в зависимости от концентрации переходного металла от нескольких единиц до нескольких сотен нанометров [5,6]. Модель Бака-Йенсена хорошо описывает магнитные свойства соединений на основе FeGe и $\mathrm{MnSi}[1,14]$, однако первопринципные расчеты для описания магнитной структуры соединения MnGe $[15,16]$ приводят к явному противоречию с экспериментальными данными 8 и, таким образом, не подтверждают применимости модели Бака-Йенсена для соединения $\mathrm{MnGe}$.

Синтез образцов моногерманидов переходных металлов является непростой задачей. Известно, что структура моногерманидов $3 d$-переходных металлов сильно зависит от числа электронов на $3 d$-уровне. Так, например, соединение $\mathrm{CrGe}$ при нормальном давлении кристаллизуется в кубической структуре $B 20$ [17], соединение $\mathrm{FeGe}$ имеет две полиморфные фазы гексагональную $B 35$ и кубическую $B 20$, а CoGe кристаллизуется в собственном структурном типе (заполненная форма структуры $\mathrm{Ni}_{3} \mathrm{Sn}_{4}$ ) [18]. Тем не менее, синтез всего ряда кубических соединений $M e \mathrm{Ge}$ со структурой типа $B 20$, а также их твердых растворов возможен, но только при высоком давлении [19]. В свою очередь, синтез этих соединений в экстремальных условиях может привести к высокой дефектности структуры образцов. Таким образом, нельзя исключить влияние дефектов кристаллографической структуры на магнитные свойства соединений. Вот почему, образцы, полученные в результате синтеза моногерманидов переходных металлов под высоким давлением, даже при условии их предварительной аттестации рентгеноструктурными методами, необходимо дополнительно исследовать методами малоуглового рассеяния для характеризации структуры на нано- и мезоскопическом масштабах от 1 до $500 \mathrm{~nm}$. Идеальным методом исследования мезоструктуры вещества является метод малоуглового рассеяния рентгеновского и/или нейтронного излучений. 
С помощью малоуглового рассеяния нейтронов или рентгеновского излучения можно оценить размеры нанообъектов, пор, зерен, кластеров, входящих в состав материала, их структурных характеристик, параметров рассеивающих поверхностей, в том числе и фрактальные размерности поверхностных и массовых фракталов. Для описания рассеяния на образцах с гладкой поверхностью и при отсутствии дефектов в объеме используется закон Порода $I(Q) \sim Q^{-n}$, где $n=4$ (при $Q>1 / R$, где $R-$ характерный масштаб рассеивающей системы, а $Q-$ переданный импульс). Отклонение показателя $n$ от 4 свидетельствует о фрактальной структуре образца. По величине $n$, в этом случае, судят о фрактальности исследуемой системы [20].

Объект называется фрактальным, если он обладает свойством самоподобия, то есть однороден на разных масштабах. Начиная с середины 80-х годов 20 века, концепция фрактала все больше используется в физике конденсированных сред [21]. В твердом теле фрактальные структуры дефектов возникают, например, при облучении кристаллов тяжелыми частицами. Важным примером являются дефектные структуры в кристаллах, подверженных интенсивной внешней нагрузке, приводящей к значительным плотностям дефектов, в результате чего проявляются коллективные эффекты. Такие эффекты приводят к включению новых структурных уровней пластической деформации. Ее носители образуют сначала фрактальные кластеры, компактизация которых приводит затем к оформлению супердефектов, являющихся структурными элементами на новом уровне [22]. Фрактальная концепция так же хорошо описывает структуры полимеров [23-27], биологических объектов [28-31], нанокластеров, пористых материалов и т.д. Движущей силой образования фрактальных структур могут быть градиенты различных физических величин: температура, давление и т. д. Например, дендрит Чернова („ветвящийся“" кристалл стали длиной около $40 \mathrm{~cm}$ ) был выращен в массивном металлическом слитке за счет градиента температуры [32].

Говоря о фрактальной мезоструктуре в твердых телах, мы подразумеваем наличие различных дефектов в материале с характерными размерами от 2 до $1000 \mathrm{~nm}$. Мезоструктура несет информацию о размере зерен, форме зерен, плотности их распределения, ориентации и т.д. Эти параметры являются очень важными для изучения физических [33] и механических [34,35] свойств материала, так как наличие или возникновение неоднородностей в структуре может повлечь за собой сильное изменение этих свойств [36]. Во многих работах (например, [37]), формирование „необычной“ мезоструктуры связывают непосредственно с методом синтеза материала.

Целью настоящей работы является исследование мезоскопической структуры образцов моногерманидов переходных металлов, синтезированных под высоким давлением [19]. Работа была мотивирована интенсивными исследованиями магнитного порядка этих материалов за последние годы [1-11]. Удивительно, что особенности мезоструктуры этих соединений до сих пор не были исследованы. Нельзя исключать, что наличие или отсутствие дефектов в кристалле, а тем более их возможная кластеризация могут существенно повлиять на магнитные свойства. Как будет показано ниже, синтезируемые соединения характеризуются наличием развитой мезоструктуры с необычным характером организации. Мы связываем появление такой мезоструктуры не со способом синтеза в камерах высокого давления, а с метастабильным состоянием соединений $\mathrm{MnGe}$ и $\mathrm{CoGe}$ и их твердых растворов при атмосферном давлении.

\section{2. Образцы и метод их синтеза}

Образцы твердых растворов квазибинарного соединения $\mathrm{Mn}_{1-x} \mathrm{Co}_{x} \mathrm{Ge}(\mathrm{c} x=0.0-1.0$, с шагом 0.1) и чистого $\mathrm{FeGe}$ с кубической структурой типа B20 были синтезированы под давлением вблизи $8 \mathrm{GPa}$. Для генерации высокого давления использовались камеры типа „тороид“ [38]. Поскольку фазовые диаграммы $\mathrm{MnGe}, \mathrm{CoGe}$ и $\mathrm{FeGe}$ сильно различаются [39-41], синтез для каждого из смешанных соединений проводился при разных температурах. Для достижения высоких температур использовался так называемый косвенный нагрев, который заключается в использовании трубчатого нагревателя $\mathrm{NaCl}$, внутри которого располагается защитный контейнер и смесь исходных компонентов. Смесь плавится пропусканием через нее электрического тока. Мощность, при которой происходит плавление, фиксируется. Затем смесь остужается до комнатной температуры и происходит кристаллизация образцов. После этого при атмосферном давлении проводился рентгенофазовый анализ (РФА) с целью определения фазового состава образцов. Выбор условий синтеза проводился по результатам РФА путем изменения мощности нагревателя при одном и том же давлении и составе. Процедура синтеза при различных условиях проводилась до тех пор, пока РФА не обнаруживал примесей в образце. Следовательно, для каждого состава подбирается свой температурный режим, при котором образец оказывается с максимальным содержанием фазы $B 20$. С этой точки зрения синтез образцов моногерманидов переходных металлов является непростой задачей - чтобы получить один образец с подавляющим количеством (около 99\%) фазы с кубической структурой типа $B 20$, предварительно проводится по 3-5 процессов синтеза.

Рентгенофазовый анализ проводился на дифрактометpe STOE IPDS-II с использованием Мо- $K_{\alpha}$ излучения при нормальном давлении и комнатной температуре. Было установлено, что полученные соединения кристаллизуются в решетку типа $B 20$.

\section{3. Результаты эксперимента}

Эксперименты с использованием малоуглового рассеяния нейтронов проводились на установке SANS-1 Цен- 
тра Майер Лейбница (MLZ, Мюнхен, Германия). Кривые малоуглового рассеяния измерялись при различных расстояниях образец-детектор $(2.2 \mathrm{~m}, 7.2 \mathrm{~m}, 20 \mathrm{~m})$ для того, чтобы перекрыть широкий диапазон переданных импульсов. Рассеянные нейтроны регистрировались в диапазоне переданных импульсов $Q$ от $0.06 \mathrm{~nm}^{-1}$ до $2.5 \mathrm{~nm}^{-1}$ с разрешением $15 \%$ от регистрируемой величины переданного импульса. Разрешение установки по переданному импульсу $\kappa_{\text {res }}$ определяется шириной прямого пучка. В наших экспериментах при максимальной длине коллимационной базы $(20 \mathrm{~m})$ и при длине волны падающих нейтронов $\lambda=0.6 \mathrm{~nm}(\Delta \lambda / \lambda=0.1)$ разрешение установки составило $\kappa_{\text {res }}=0.006 \mathrm{~nm}^{-1}$. С целью разделить магнитное и ядерное рассеяние, температура образца варьировалась в диапазоне от $T=3.0 \mathrm{~K}$ до $T=320.0 \mathrm{~K}$ с точностью $0.1 \mathrm{~K}$. Измерения проводились в нулевом магнитном поле. В ходе эксперимента по 3 образца помещались кассету, которая устанавливалась в криостате, после чего кассета с образцами охлаждалась до необходимых при исследовании температур. Для того, чтобы рассеяние происходило только на одном из образцов, пространство между ними в кассете заполнялось поглощающим нейтроны кадмием.

Поскольку образцы, полученные в результате синтеза, являются поликристаллическими, рассеяние нейтронов считалось изотропным по азимутальному углу. Поэтому для улучшения статистики проводилось азимутальное усреднение интенсивности рассеяния. Зависимость интенсивности рассеяния нейтронов от переданного импульса $\mathrm{I}(\mathrm{Q})$ для образца чистого $\mathrm{MnGe}$ представлена на рис. 1 и 2 для температур $T=3 \mathrm{~K}$ и $T=317 \mathrm{~K}$ соответственно. Экспериментальные зависимости были аппроксимированы выражением следующего вида:

$$
I(Q)=B /\left(Q^{2}+\kappa^{2}\right)^{n / 2}+I_{b g}+I_{m}(Q)
$$

где $B$ - амплитуда когерентного рассеяния на неоднородностях мезоструктуры; дробная степень $n$ связана с фрактальной размерностью образца; $I_{b g}-$ это интенсивность некогерентного фона немагнитной природы; $I_{m}-$ магнитный вклад в рассеяние. В данном случае $\kappa$ связан либо с разрешением установки $\kappa_{\text {res}}$, либо с размером неоднородности с корреляционным радиусом $\xi=2 \pi / \kappa$. В случае, если $\xi<2 \pi / \kappa_{\text {res }}$, то $\kappa=2 \pi / \xi$, что позволяет измерить размер неоднородности $\xi$. Если $\xi>2 \pi / \kappa_{\text {res }}$, то $\kappa=\kappa_{\text {res }}$, и поэтому $\kappa$ является параметром, ограничивающим возможность измерить верхнюю границу фрактала и может сама по себе служить оценочным значением, ограничивающим верхнюю границу фрактала снизу.

Форма зависимости магнитного рассеяния от переданного импульса подробно изучена в работе [8]. $\mathrm{B}$ настоящей работе вклад от магнитной структуры рассматривается в качестве паразитного и учитывается с целью более точного описания рассеяния на мезоструктуре поликристалла. При температуре $T=3 \mathrm{~K}$ (рис. 1) магнитное рассеяние $I_{m}$ представляет собой брэгговский

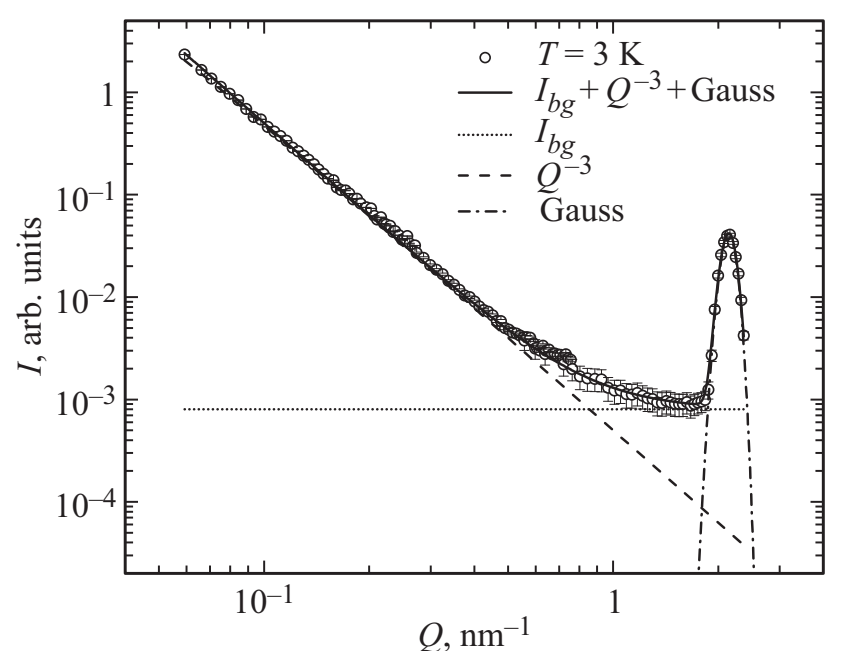

Рис. 1. Зависимость интенсивности малоуглового рассеяния нейтронов от переданного импульса для MnGe при температуpe $3 \mathrm{~K}$.

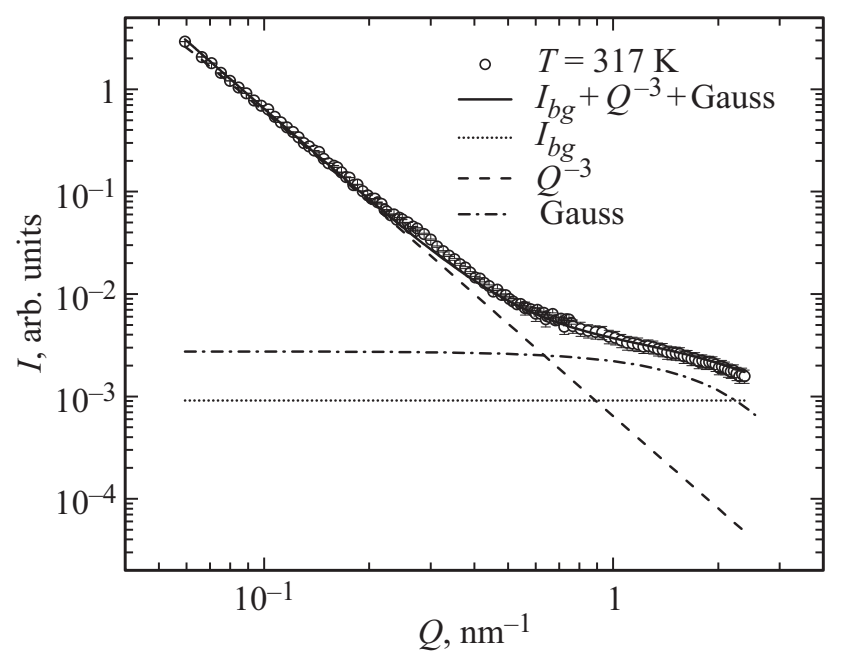

Рис. 2. Зависимость интенсивности малоуглового рассеяния нейтронов от переданного импульса для MnGe при температуpe $317 \mathrm{~K}$.

рефлекс, связанный с рассеянием на магнитной геликоидальной структуре. Форма данного пика хорошо аппроксимируется функцией Гаусса с центром при $Q=k_{s}$, где $k_{s}$ - это волновой вектор спирали. Магнитный вклад также наблюдается при температуре $T=317 \mathrm{~K}$ (рис. 2). Он определяется наличием ферромагнитных нано-областей и аппроксимируется функцией Гаусса с центром при $Q=0$ [8]. Учет и вычитание магнитного вклада в рассеяние в итоге позволили определить параметры функции рассеяния на мезоструктуре: амплитуды когерентного и некогерентного рассеяния $\left(B\right.$ и $\left.I_{b g}\right)$, а также показатель $n$ в выражении для $Q$-зависимости интенсивности рассеяния (1). Все эти параметры не зависят от температуры. Параметр $\kappa$ для всех аппроксимаций равнялся нулю с точностью до ширины функции 
разрешения $\kappa_{\text {res }}$. Следовательно, можно дать оценку снизу на верхнюю границу фрактальной неоднородности мезоструктуры $\xi=2 \pi / \kappa$, которая равна $1 \mu \mathrm{m}$. Показатель степени $n$ в зависимости интенсивности рассеяния от переданного импульса $I(Q)$ равнялся $3.00 \pm 0.02$ для всего диапазона температур для образца чистого $\mathrm{MnGe}$. Принимая во внимание, что $I_{b g}$ также мало, можно заключить, что интенсивность рассеяния нейтронов $I(Q)$ пропорциональна $Q^{-3}$ (рис. 1 и 2).

Аналогично аппроксимации данных малоуглового рассеяния в чистом $\mathrm{MnGe}$ была аппроксимирована вся совокупность данных для квазибинарных соединений $\mathrm{Mn}_{1-x} \mathrm{Co}_{x} \mathrm{Ge}$ для различных концентраций $x$. На рис. 3 представлена зависимость интенсивности рассеяния от переданного импульса $I(Q)$ для соединений $\mathrm{Mn}_{1-x} \mathrm{Co}_{x} \mathrm{Ge}$ для различных концентраций $x$ при $T=300 \mathrm{~K}$. Как видно из рисунка, зависимости $I(Q)$ для смешанных соединений $\mathrm{Mn}_{1-x} \mathrm{Co}_{x} \mathrm{Ge}$, построенные в двойном логарифмическом масштабе, расположены параллельно друг другу, демонстрируя одинаковый с MnGe закон рассеяния: $I(Q)$ пропорциональна $Q^{-3}$.

На рис. 4 представлена зависимость показателя степени $n$ в зависимости интенсивности рассеяния от переданного импульса $I(Q)$ от концентрации $x$ для всего ряда соединений $\mathrm{Mn}_{1-x} \mathrm{Co}_{x} \mathrm{Ge}$. Как видно из рисунка, показатель степени $n$ не меняется с концентрацией. Можно сделать вывод, что концентрация Со не влияет форму рассеивающих неоднородностей в мезоструктуре образцов соединений $M n_{1-x} \mathrm{Co}_{x} \mathrm{Ge}$.

Следует отметить, что наблюдаемая для образцов $\mathrm{MnGe}$ форма зависимости интенсивности малоуглового рассеяния от переданного импульса $(I(Q)$ пропорциональна $Q^{-3}$ ) является совершенно необычной. В экспериментах малоуглового рассеяния нейтронов в материалах с фрактальной структурой наблюдается степенная зависимость интенсивности рассеяния от переданного импульса вида $I(Q) \sim\left(1+(Q \xi)^{2}\right)^{-n / 2}(n<4)$. Для объемных или массовых фракталов $n$ совпадает с фрактальной размерностью $D_{v}$, причем $1<D_{v}<3$. Для рассеяния от трехмерных объектов с фрактальной поверхностью $3<n=6-D_{s}<4, D_{s}-$ фрактальная размерность поверхности $2<D_{S}<3$. При этом корреляционная функция такого фрактального объекта имеет вид степенной функции расстояния $(r / \xi)^{D_{v}-3}$, где $\xi-$ это размер частицы [20]. Пограничный случай перехода от объемного к поверхностному фракталу, соответствующий кубической зависимости сечения рассеяния нейтронов $\left(1+(Q \xi)^{2}\right)^{-3 / 2}$, приводит к корреляционной функции, асимптотика которой в области фрактального поведения при $r / \xi<1$ пропорциональна $\ln (r / \xi)$ [42]. Это соответствует особому типу самоподобия с аддитивным законом масштабирования, а не мультипликативным, как в случае объемного фрактала.

Так, например, совершенно иное, по сравнению с образцами $\mathrm{MnGe}$, рассеяние наблюдается на образцах FeGe. Следует отметить, что образцы FeGe также были синтезированы под высоким давлением в камерах типа

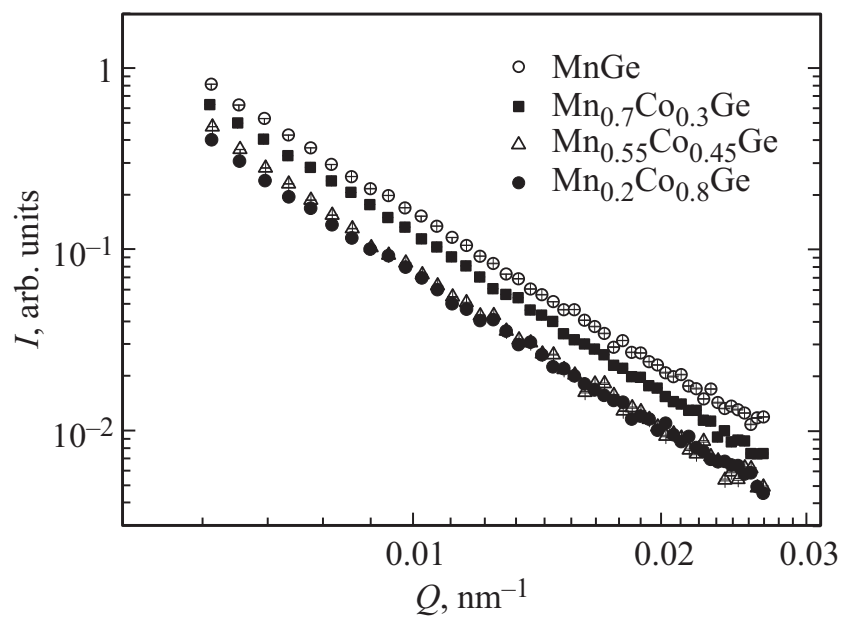

Рис. 3. Зависимость интенсивности малоуглового рассеяния нейтронов от переданного импульса для соединений $\mathrm{Mn}_{1-x} \mathrm{Co}_{x} \mathrm{Ge}$ при $x=0.0,0.3,0.45,0.8$ при температуре $T=300 \mathrm{~K}$.

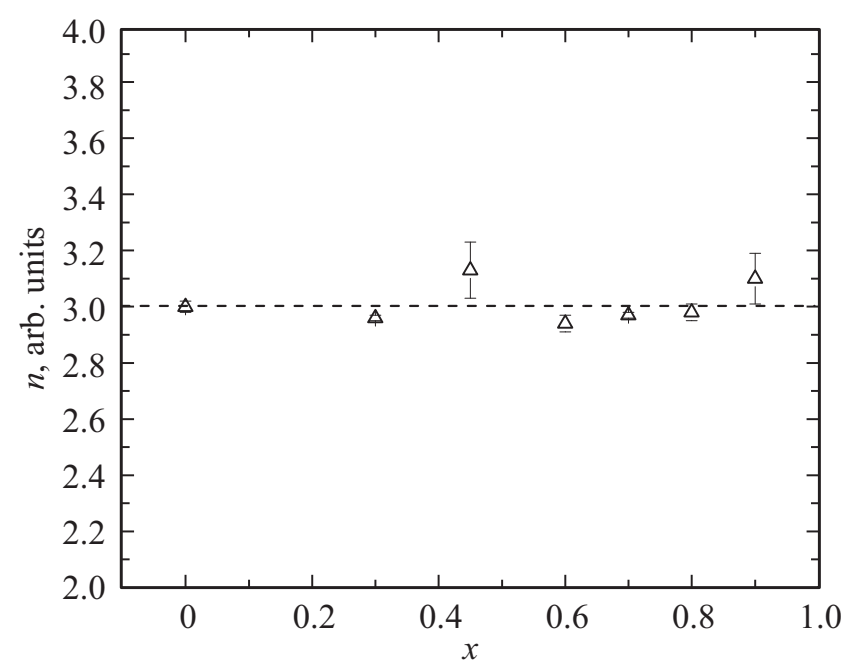

Рис. 4. Показатель степени $n$ в зависимости интенсивности малоуглового рассеяния нейтронов от переданного импульса $I(Q)$ от концентрации твердого раствора $x$ в соединениях $\mathrm{Mn}_{1-x} \mathrm{Co}_{x} \mathrm{Ge}$. Штрихованная линия соответствует $n=3$.

„тороид“ $[19,38]$. На рис. 5 представлена зависимость интенсивности малоуглового рассеяния нейтронов для FeGe (при $T=290 \mathrm{~K}$ ). Экспериментальная зависимость для $\mathrm{FeGe}$ была также аппроксимирована выражением (1). Показатель степени $n$ в зависимости интенсивности рассеяния от переданного импульса $I(Q)$ был равен $4.1 \pm 0.1$. То есть наблюдается рассеяние нейтронов однородными частицами с гладкой поверхностью (асимптотика Порода) [20,42].

Таким образом, малоугловое рассеяние нейтронов на образце FeGe показало, что образец состоит из однородных по плотности кристаллитов с резкой границей. Это указывает на отсутствие какой-либо мезоструктуры внутри кристаллитов FeGe. Эксперименты МУРН 


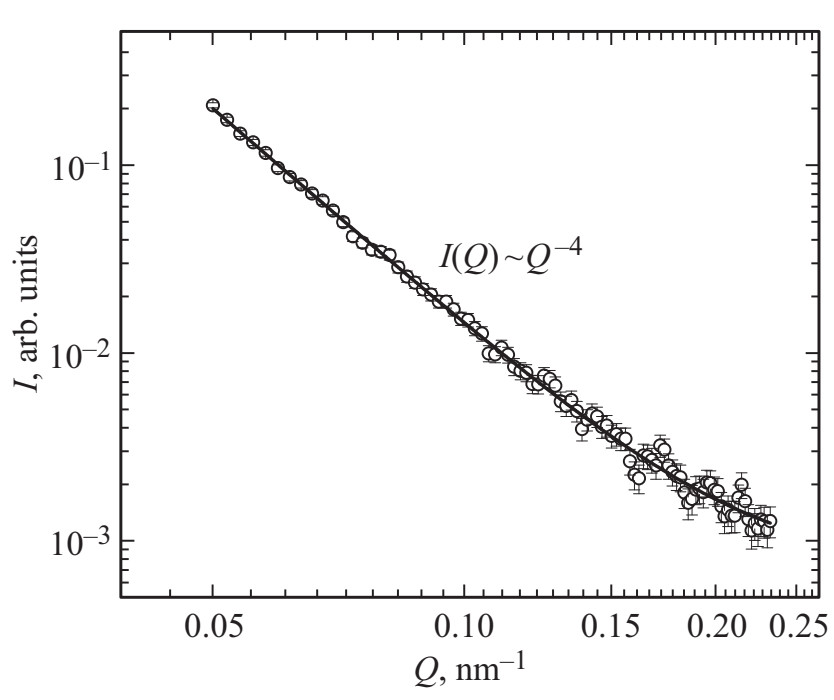

Рис. 5. Зависимость интенсивности малоуглового рассеяния нейтронов от переданного импульса для FeGe при температуре $290 \mathrm{~K}$.

на образцах $\mathrm{Mn}_{1-x} \mathrm{Co}_{x} \mathrm{Ge}$, напротив, показывают, что образцы состоят из кристаллитов, внутри которых находится развитая мезоструктура с характерной организацией неоднородностей, описываемой корреляционной функцией с логарифмической зависимостью плотности от расстояния $r$. Поскольку и образцы $\mathrm{FeGe}$, и образцы $\mathrm{Mn}_{1-x} \mathrm{Co}_{x} \mathrm{Ge}$ были синтезированы с использованием одинаковой техники, мы не связываем появление такой мезоструктуры со способом синтеза в камерах высокого давления. Предполагается, что такая мезоструктура представляет собой фрактальную структуру дефектов в кристаллитах MnGe и $\mathrm{CoGe}$ и их твердых растворов, которая обусловлена в первую очередь метастабильным состоянием этих соединений при атмосферном давлении [19], что отличает их от устойчивого соединения $\mathrm{FeGe}$.

\section{4. Обсуждение результатов}

Как показано в [21,22], дефекты сами по себе являются самостоятельными структурными образованиями, обладающими присущими им свойствами, например, геометрической конфигурацией, распределением упругих полей и т.д. В рамках такой концепции система дефектов эргодична и со временем занимает все выделенное ей фазовое пространство независимо друг от друга, и в этом случае отсутствует какая-либо иерархическая соподчиненность в поведении дефектов. При такой постановке задачи зависимость системы дефектов от конфигурационных координат имеет вид регулярного распределения минимумов, наименьший из которых соответствует стабильному состоянию, все остальные метастабильному.

Однако, если плотность дефектов достигает высоких значений, то проявляются коллективные эффекты в их поведении. Это означает установление когерентной связи в ансамбле дефектов типа той, что обуславливает фазовые и кинетические превращения. В то же время установление когерентной связи в ансамбле дефектов одного структурного уровня приводит к автолокализованному образованию, играющему роль исходного структурного элемента на более высоком уровне. В частности, автолокализованный характер продуктов этого превращения (супердефектов) является следствием происходящей в результате превращения потери эргодичности, которая, в свою очередь, связана с иерархической соподчиненностью. Последняя выражается в том, что супердефект образуется в результате когерентной связи дефектов на нижнем структурном уровне. Поскольку на зависимости термодинамического потенциала от конфигурационных координат исходным дефектам отвечают более узкие минимумы, чем супердефектам, то установление иерархической соподчиненности приводит к фрактальной структуре распределения термодинамического потенциала в конфигурационном пространстве [21,22].

Как показывают примеры спиновых стекол [43], фрактальность структуры распределения термодинамического потенциала коренным образом изменяет ее термодинамические и кинетические свойства. Это обусловлено разбиением конфигурационного пространства на множество областей (долин или компонент), каждой из которых отвечает свой статистический ансамбль. В результате определение средних производится в два этапа: сначала усреднением по чистому ансамблю данной долины, а затем - по ансамблю долин. Кинетический аспект поведения такой системы, обусловленный слабым восстановлением эргодичности, определяется медленным объединением долин в кластеры более крупных компонент. Данный процесс удобно представить как движение по узлам иерархического дерева Кейли, отвечающим долинам, к его стволу. Такое представление конфигурационного пространства дефектов посредством ультраметрической топологии было в предложено в работах $[21,22]$.

Важность концепции ультраметрического пространства обусловлена тем обстоятельством, что отражая иерархическую структуру системы, оно реализует так называемую логарифмическую метрику для физически наблюдаемых величин. Это означает, что в таком пространстве расстояние $l$ оказывается линейной функцией логарифма наблюдаемой величины $r$. Поскольку манипулирование с $\ln (r)$ менее удобно, чем с линейной зависимостью от $l$, то вместо обычной оси значений удобно ввести соответствующее ультраметрическое пространство, характеризуемое расстоянием $l$, и все выкладки проводить в этом пространстве $[44,45]$.

Как показано в работе [42], обнаруженный в этой работе случай кубической зависимости сечения рассеяния нейтронов от переданного импульса $\left(1+(\xi Q)^{2}\right)^{-3 / 2}$ приводит к корреляционной функции, асимптотика которой в области фрактального поведения при $r / \xi<1$ пропорциональна $\ln (r / \xi)$. По существу, корреляционная 
функция дефектов в кристаллите и является метрикой для данной системы, а тот факт, что она является логарифмической делает этот случай особенным в том смысле, что характеризует эту систему как иерархическую фрактальную систему, описываемую ультраметрическим пространством состояний, которое хорошо визуализуется деревом Кейли $[21,22]$.

\section{5. Заключение}

Обнаруженные особенности внутреннего строения образцов являются следствием внутреннего энергетического ландшафта кристаллической структуры, а не кристаллизации образцов в экстремальных условиях, то есть при высокой температуре и в высоком давлении. Следует отметить, что образец $\mathrm{FeGe}$, который является стабильным при нормальном давлении, характеризуется функцией рассеяния и соответственно корреляционной функцией, которые подразумевают однородное распределение плотности вещества внутри кристаллитов с резкими границами. В то же время внутри кристаллитов в образцах $\mathrm{Mn}_{1-x} \mathrm{Co}_{x} \mathrm{Ge}$ возникают дефекты с пространственной организацией, описываемой фрактальной моделью с логарифмической зависимостью парной корреляционной функции. Нами выдвинута гипотеза, что при нормальном давлении в соединениях $\mathrm{Mn}_{1-x} \mathrm{Co}_{x} \mathrm{Ge}$ атомы Me/Ge вытесняются из решетки $B 20$ на поверхность. Дефицит атомов $\mathrm{Me} / \mathrm{Ge}$ формирует структуру в виде логарифмического фрактала, который хорошо визуализуется деревом Кейли. Такой вид фрактала соответствует особому типу самоподобия с аддитивным законом масштабирования, а не мультипликативным, как в случае объемного фрактала [42].

\section{Список литературы}

[1] B. Lebech, J. Bernhard, T. Freltoft. J. Phys.: Condens. Matter 1, 6105 (1989).

[2] N. Kanazawa, Y. Onose, T. Arima, D. Okuyama, K. Ohoyama, S. Wakimoto, K. Kakurai, S. Ishiwata, Y. Tokura. Phys. Rev. Lett. 106, 156603 (2011).

[3] O.L. Makarova, A.V. Tsvyashchenko, G. Andre, F. Porcher, L.N. Fomicheva, N. Rey, I. Mirebeau. Phys. Rev. B 85, 205205 (2012).

[4] N. Kanazawa, J.-H. Kim, D.S. Inosov, J.S. White, N. Egetenmeyer, J.L. Gavilano, S. Ishiwata, Y. Onose, T. Arima, B. Keimer, Y. Tokura. Phys. Rev. B 86, 134425 (2012).

[5] S.V. Grigoriev, N.M. Potapova, S.-A. Siegfried, V.A. Dyadkin, E.V. Moskvin, V. Dmitriev, D. Menzel, C.D. Dewhurst, D. Chernyshov, R.A. Sadykov, L.N. Fomicheva, A.V. Tsvyashchenko. Phys. Rev. Lett. 110, 207201 (2013).

[6] K. Shibata, X.Z. Yu, T. Hara, D. Morikawa, N. Kanazawa, K. Kimoto, S. Ishiwata, Y. Matsui, Y. Tokura. Nat. Nanotech. 8, 723 (2013).

[7] M. Deutsch, P. Bonville, A.V. Tsvyashchenko, L.N. Fomicheva, F. Porcher, F. Damay, S. Petit, I. Mirebeau. Phys. Rev. B 90, 144401 (2014).
[8] E. Altynbaev, S.-A. Siegfried, V. Dyadkin, E. Moskvin, D. Menzel, A. Heinemann, C. Dewhurst, L. Fomicheva, A. Tsvyashchenko, S. Grigoriev. Phys. Rev. B 90, 174420 (2014).

[9] E.V. Altynbaev, A.S. Sukhanova, S.-A. Siegfried, V.A. Dyadkin, E.V. Moskvin, D. Menzel, A. Heinemann, A. Schrayer, L.N. Fomicheva, A.V. Tsvyashchenko, S.V. Grigoriev. J. Surf. Investigation 10, 777 (2016).

[10] N. Martin, M. Deutsch, F. Bert, D. Andreica, A. Amato, P. Boni, R. De Renzi, U.K. Rossler, P. Bonville, L.N. Fomicheva, A.V. Tsvyashchenko, I. Mirebeau. Phys. Rev. B 93, 174405 (2016).

[11] E. Altynbaev, S.-A. Siegfried, E. Moskvin, D. Menzel, C. Dewhurst, A. Heinemann, A. Feoktystov, L. Fomicheva, A. Tsvyashchenko, S. Grigoriev. Phys. Rev. B 94, 174403 (2016).

[12] O. Nakanishi, A. Yanase, A. Hasegawa, M. Kataoka. Solid State Commun. 35, 995 (1980).

[13] P. Bak, M.H. Jensen. J. Phys. C 13, L881 (1980).

[14] S.V. Grigoriev, S.V. Maleyev, A.I. Okorokov, Y.O. Chetverikov, P. Böni, R. Georgii, K. Pranzas. Phys. Rev. B, 74, (21), 214414, (2006).

[15] T. Koretsune, N. Nagaosa, R. Arita. Sci. Rep. 5, 13302 (2015).

[16] J. Gayles, F. Freimuth, T. Schena, G. Lani, P. Mavropoulos, R. Duine, S. Blugel, J. Sinova, Y. Mokrousov. Phys. Rev. Lett. 115, 036602 (2015).

[17] M. Hansen. Mc-Graw-Hill Book Company, 2nd ed. (1958). $532 \mathrm{p}$.

[18] W.B. Pearson, A Handbook of Lattice Spacings and Structures of Metals and Alloys. V. 1, Pergamon, Oxford (1958) 185 p.

[19] A.V. Tsvyashchenko. J. Less-Common Met. 99, L9 (1984).

[20] J. Teixera. On Growth and Form-Fractal and Non-Fractal Pattern in Physics / Eds H.E. Stanley, N. Ostrovsky. Martinus Nijloff Publ., Boston (1986) 145 p.

[21] А.И. Олемской, А.Я. Флат. УФН, 162, 12, 1 (1993).

[22] А.И. Олемской, И.А. Скляр. УФН. 162, 6, 29 (1992).

[23] D.W. Maccarthy, J.E. Mark, D.W. Schaefer. J. Polymer Sci. B 36, 1167 (1998).

[24] W. Dale, R.S. Justic. Macromolecules, 24, 40 (2007).

[25] R.J. Roe. Methods of X-ray and Neutron Scattering in Polymer Science, Oxford University Press, N.Y. (2000).

[26] Harold D. Bale, Paul. Phys. Rev. L, 53, 6, 596 (1984).

[27] A.J. Hurd, D.W. Schaefer, J.E. Martin. Phys. Rev. A 35, 52361 (1987).

[28] A. Ilatovskiy, D. Lebedev, M. Filatov, M. Petukhov, V. IsaevIvanov. J. Phys. Conf. Ser. 351, 012007 (2012).

[29] A. Bancaud, C. Lavelle, S. Huet, J. Ellenberg. Nucleic Acids Res. 40, 18, 878392 (2012).

[30] P.W. Schmidt. The Fractal Approach to Heterogeneous Chemistry: Surfaces, Colloids, Polymers / Ed D. Avnir. Ch. 2.2 (1989). P. 67-79.

[31] D.V. Lebedev, M.V. Filatov, A.I. Kuklin, A.K. Islamov, E. Kentzinger, R.A. Pantina, B.P. Toperverg, V.V. Isaev-Ivanov. FEBS Lett. 579, 1465 (2005).

[32] Ю.Д. Третьяков. Дендриты, фракталы и материалы. Соросовский образовательный журнал 1196 (1998).

[33] G.P. Kopitsa, A.E. Baranchikov, O.S. Ivanova, A.D. Yapryntsev, S.V. Grigoriev, K. Pranzas, V.K. Ivanov. J. Phys. Conf. Ser. 340, 012 (2012).

[34] В.В. Поляков, С.В. Кучерявский, А.В. Егоров. Физическая мезомеханика 5, 103 (2001). 
[35] П.В. Кузнецов, И.В. Петракова. Физическая мезомеханика S1-1, 389 (2004).

[36] F. Marlow. J. Mater. Chem. J. Mater. Chem 17, 2168 (2007).

[37] П.В. Кузнецов, В.Е. Панин, К.В. Левин. Физическая мезомеханика, 3, 89 (2000).

[38] L.G. Khvostantsev, V.N. Slesarev, V.V. Brazhkin. High Press. Res. 24, 371 (2004).

[39] E. Arras, D. Caliste, T. Deutsch, F. Lançon, P. Pochet. Phys. Rev. B 83, 17, 174103 (2011).

[40] E.I. Tonkov. High pressure phase transformations: A handbook CRC Press. (1992). V. 2.

[41] Y.H. Zhuang, X. Chen, J.L. Yan, R.F. Li, C.H. Ma. J. Alloys Comp. 465, 1, 216 (2008).

[42] Е.Г. Яшина, С.В. Григорьев. Поверхность. 8, 3 (2017).

[43] В.С. Доценко. УФН 163, 6, 1 (1993).

[44] R. Rammal, G. Toulouse, V.A. Virasoro. Rev. Mod. Phys. 58, 765 (1986).

[45] С.Л. Гинзбург. Необратимые явления в спиновых стеклах. Наука, М. (1989). 